EESTI NSV TEADUSTE AKADEEMIA TOIMETISED. 25. KOIDE FOOSIKA * MATEMAATIKA. 1976, NR. 1

ИЗВЕСТИЯ АКАДЕМИН НАУК ЭСТОНСКОП ССР. ТОМ 25 ФИЗИКА * МАТЕМАТНКА. 1976, № 1

\title{
НОВЫЙ ПРИБЛИЖЕННЫЙ МЕТОД АНАЛИЗА НЕОДНОРОДНОГО ОПТИЧЕСКОГО СЛОЯ
}

Развит метод приближенного вычисления коэффициента отражения света на неоднородном оптическом слое, основанный на аппроксимации обратной величины показателя преломления квадратичной функцией координаты.

\section{Введение}

Под анализом оптического слоя мы понимаем здесь, как обычно, нахождение по заданной толщине и показателю преломления слоя его оптических характеристик в зависимости от длины волны. В случае неоднородного слоя показатель преломления $n(z)$ задается как функция координаты $z$ внутри слоя. Задача сводится, в предположении нормального падения света, к решению одномерного волнового уравнения

$$
d^{2} U / d z^{2}+k^{2} n^{2}(z) U=0,
$$

где $k$ - волновое число. Случай наклонного падения света тоже может быть приведен к уравнению того же вида, только под $n(z)$ следует понимать эффективный показатель преломления [ $\left.{ }^{1}\right]$. В дальнейшем будем для простоты считать падение нормальным. Зная линейно независимые решения уравнения (1), можно, как показано в [ $\left.{ }^{1}\right]$, найти матрицу интерференции слоя, определяющую все его оптические свойства.

Однако уравнение (1) решается в замкнутом виде только в сравнительно немногих частных случаях. Отсюда возникает актуальная задача приближенного решения этого уравнения. В дополнение к существующим приближенным методам мы предложим в настоящей статье еще один - метод квадратичной аппроксимации, проиллюстрировав его двумя примерами и проведя сравнение с методом, развитым в статьях $[2,3]$.

\section{Метод квадратичной аппроксимации}

Как показано в $\left[{ }^{4}\right]$, одним из случаев, когда уравнение (1) имеет замкнутое решение, является квадратичная зависимость обратной величины показателя преломления:

$$
n^{-1}(z)=A z^{2} / h^{2}+2 B z / h+C .
$$


Здесь $h$ - толщина слоя, $A, B, C$ - безразмерные постоянные. В $\left[{ }^{4}\right]$ проведено подробное решение волнового уравнения для этого случая и получены формулы для матрицы интерференции. Этим решением можно воспользоваться для приближенного решения в случае произвольного вида функции $n(z)$. Достаточно предположить, что $n^{-1}(z)$ не имеет внутри слоя точек перегиба. Тогда мы можем заменить эту функцию приближенно квадратичной функцией вида (2), выбрав значения коэффициентов $A, B, C$ так, чтобы значения приближенной функции $n^{-1}(z)$ совпадали в трех точках (напр., на краях и в середине слоя) с точными значениями заданной функции $n^{-1}(z)$. Если же $n^{-1}(z)$ имеет внутри слоя точки перегиба, то следует разделить слой в этих точках на несколько частей и применять затем тот же метод для каждой части отдельно. А так как матрица интерференции всей совокупности равна произведению матриц интерференции отдельных частей, то расчет может вестись, как и при отсутствии точек перегиба.

Конкретно выберем два таких вида функции $n(z)$, для которых существует точное решение волнового уравнения. Это позволит нам провести сравнение результатов точного и приближенного решения. Оба вида $n(z)$ заимствованы из перечня случаев, допускающих точное решение, приведенного в монографии [ $\left.{ }^{5}\right]$ (с. $\left.129-131\right)$. В обоих этих примеpax $n(z)$ вещественно. Поэтому достаточно вычислять только коэффициент отражения.

\section{Обозначения и общие формулы}

Обозначим границы слоя через $z_{1}$ и $z_{2}$ и значения показателя преломления на границах через $n_{1}$ и $n_{2}$, т. е.

$$
\begin{aligned}
& n\left(z_{1}\right)=n_{1}, \\
& n\left(z_{2}\right)=n_{2} .
\end{aligned}
$$

Показатели преломления ограничивающих сред обозначим в общих формулах через $n_{1}^{0}$ и $n_{2}^{0}$. Все численные расчеты проведем, однако, для случая, когда

$$
\begin{aligned}
& n_{1}^{0}=n_{1}, \\
& n_{2}^{0}=n_{2},
\end{aligned}
$$

т. е. когда на границах слоя показатель преломления изменяется непрерывно. Толщину слоя, как и в формуле (2), обозначим через $h$ :

$$
h=z_{2}-z_{1} \text {. }
$$

Введем безразмерную спектральную переменную

$$
\alpha=h k \text {. }
$$

Используем также введенные в $\left[{ }^{6}\right]$ обозначения

$$
G(v)=\left(\begin{array}{ll}
\operatorname{ch} v & \operatorname{sh} v \\
\operatorname{sh} v & \operatorname{ch} v
\end{array}\right)
$$

и

$$
v=\frac{1}{2} \ln n
$$

Матрица интерференции слоя имеет следующий общий вид:

$$
L_{12}^{0}=G\left(v_{1}^{0}\right)\left(\begin{array}{ll}
l_{0}+l_{3} & l_{1}-i l_{2} \\
l_{1}+i l_{2} & l_{0}-l_{3}
\end{array}\right) G\left(-v_{2}^{0}\right),
$$


где $l_{0}, l_{1}, l_{2}, l_{3}$ выражаются через линейно независимые решения волнового уравнения (см. $\left[{ }^{1}\right]$, формулы (26), (31) и (32)).

\section{Первый вид показателя преломления}

Пусть

$$
n(z)=\left[n_{1}^{2} z_{2} / h-n_{2}^{2} z_{1} / h+\left(n_{2}^{2}-n_{1}^{2}\right) z / h\right]^{1 / 2} .
$$

Волновое уравнение

$$
d^{2} U / d z^{2}+k^{2} h^{-1}\left[n_{1}^{2} z_{2}-n_{2}^{2} z_{1}+\left(n_{2}^{2}-n_{1}^{2}\right) z\right] U=0
$$

подстановкой

$$
y=-\beta^{2} n^{2}(z),
$$

где для краткости положено

$$
\beta=\left[\alpha\left(n_{2}^{2}-n_{1}^{2}\right)^{-1}\right]^{1 / 3},
$$

приводится к уравнению Эйри

$$
d^{2} U / d y^{2}-y U=0 .
$$

В качестве линейно независимых решений этого уравнения примем функции Эйри $u(y)$ и $v(y)$ (см., напр., таблицы $\left.\left[{ }^{7}\right]\right)$, удовлетворяющие условию

$$
u^{\prime} v-u v^{\prime}=1
$$

где штрих означает производную по $y^{*}$. Обозначив линейно независимые решения уравнения (10) через $U_{1}$ и $U_{2}$, имеем:

$$
\begin{aligned}
& U_{1}=u\left(-\beta^{2} n^{2}(z)\right), \\
& U_{2}=v\left(-\beta^{2} n^{2}(z)\right) .
\end{aligned}
$$

Теперь мы можем по схеме статьи [ $\left.{ }^{1}\right]$ вычислить матрицу интерференции. Для величин $l_{0}, l_{1}, l_{2}, l_{3}$ находим следующие выражения:

$$
\begin{aligned}
& l_{0}=\frac{1}{2}\left(H_{1}-H_{2}\right), \\
& l_{1}=-\frac{1}{2}\left(H_{1}+H_{2}\right), \\
& l_{2}=\frac{1}{2}\left(\beta H-\beta^{-1} H_{12}\right), \\
& l_{3}=\frac{i}{2}\left(\beta H+\beta^{-1} H_{12}\right),
\end{aligned}
$$

где

$$
\begin{aligned}
& H_{1}=u_{1}^{\prime} v_{2}-u_{2} v^{\prime}{ }_{1}, \\
& H_{2}=u_{1} v^{\prime}{ }_{2}-u^{\prime}{ }_{2} v_{1}, \\
& H=u_{1} v_{2}-u_{2} v_{1}, \\
& H_{12}=u_{1}^{\prime}{ }_{1} v^{\prime}{ }_{2}-u^{\prime}{ }_{2} v^{\prime}{ }_{1},
\end{aligned}
$$

а индексы у $u$ и $v$ и их производных указывают на значения аргумента $z=z_{1}$ и $z=z_{2}$, например, $u_{1,2}=u\left(-\beta^{2} n_{1,2}^{2}\right) \quad$ и т. д. Отметим, что величины $H_{1}, H_{2}, H$ и $H_{12}$ удовлетворяют соотношению

$$
\mathrm{HH}_{12}-\mathrm{H}_{1} \mathrm{H}_{2}=1 \text {. }
$$

* Обозначение $v$ функции Эйри совпадает с обозначением $\frac{1}{2} \ln n$ в формуле (8). Однако смешение этих величин в нижеследующем практически исключено. 
Подставляя выражения $l_{0}, l_{1}, l_{2}, l_{3}$ из (17) в формулу (9), учитывая формулы (7) и (8) и перемножая матрицы, находим:

$$
L_{12}^{0}=\frac{1}{2 \sqrt{v_{1}^{0} v_{2}^{0}}}\left(\begin{array}{cc}
v_{2}^{0} H_{1}-v_{1}^{0} H_{2}+ & -v_{2}^{0} H_{1}-v_{1}^{0} H_{2}- \\
+i\left(v_{1}^{0} v_{2}^{0} H+H_{12}\right) & -i\left(v_{1}^{0} v_{2}^{0} H-H_{12}\right) \\
-v_{2}^{0} H_{1}-v_{1}^{0} H_{2}+ & v_{2}^{0} H_{1}-v_{1}^{0} H_{2}- \\
+i\left(v_{1}^{0} v_{2}^{0} H-H_{12}\right) & -i\left(v_{1}^{0} v_{2}^{0} H+H_{12}\right)
\end{array}\right),
$$

где

$$
\begin{aligned}
& v_{1}^{0}=\beta n_{1}^{0}, \\
& v_{2}^{0}=\beta n_{2}^{0} .
\end{aligned}
$$

В случае непрерывности показателя преломления на границах слоя в формуле $(20)$ следует $v_{1}^{0}$ и $v_{2}^{0}$ заменить на

$$
\begin{aligned}
& v_{1}=\beta n_{1}, \\
& v_{2}=\beta n_{2} .
\end{aligned}
$$

Энергетический коэффициент отражения выражается в этом последнем случае формулой

$$
R_{12}=\frac{\left(v_{2} H_{1}+v_{1} H_{2}\right)^{2}+\left(v_{1} v_{2} H-H_{12}\right)^{2}}{\left(v_{2} H_{1}-v_{1} H_{2}\right)^{2}+\left(v_{1} v_{2} H+H_{12}\right)^{2}} .
$$

\section{Второй вид показателя преломления}

Пусть

причем

$$
n(z)=\left(m^{2}-\mu^{2} h^{2} / z^{2}\right)^{1 / 2},
$$

$$
\begin{aligned}
& m^{2}=\frac{n_{2}^{2} z_{2}^{2}-n_{1}^{2} z_{1}^{2}}{z_{2}^{2}-z_{1}^{2}}, \\
& \mu^{2}=\frac{\left(n_{2}^{2}-n_{1}^{2}\right) z_{1}^{2} z_{2}^{2}}{h^{2}\left(z_{2}^{2}-z_{1}^{2}\right)} .
\end{aligned}
$$

Волновое уравнение

подстановкой

$$
d^{2} U / d z^{2}+k^{2}\left(m^{2}-\mu^{2} h^{2} / z^{2}\right) U=0
$$

$$
\begin{aligned}
& U=y^{1 / 2} Y(y), \\
& y=k m z
\end{aligned}
$$

приводится к уравнению Бесселя $p$-го порядка

где

$$
d^{2} Y / d y^{2}+y^{-1} d Y / d y+\left(1-p^{2} / y^{2}\right) Y=0,
$$

$$
p=\left(\frac{1}{4}+\mu^{2} \alpha^{2}\right)^{1 / 2} \text {. }
$$

Следовательно, линейно независимые решения уравнения (26) суть:

$$
\begin{aligned}
& U_{1}(z)=(k z)^{1 / 2} J_{p}(k m z), \\
& U_{2}(z)=(k z)^{1 / 2} N_{p}(k m z),
\end{aligned}
$$

где $J_{p}$ и $N_{p}$ - функции Бесселя 1 -го и 2 -го рода $p$-го порядка. Отсюда 
опять по схеме статьи $\left[{ }^{1}\right]$ вычисляем матрицу интерференции. Величины $l_{0}, l_{1}, l_{2}, l_{3}$ в этом случае выражаются формулами:

$$
\begin{aligned}
& l_{0}=-\frac{\operatorname{sh}^{2} \zeta}{m \alpha} \cdot S+\frac{S_{2}-S_{1}}{2} \\
& l_{1}=\frac{\operatorname{sh} \zeta \operatorname{ch} \zeta}{m \alpha} \cdot S+\frac{S_{2}+S_{1}}{2} \\
& l_{2}=\frac{1}{2 m}\left(1-\frac{\operatorname{sh}^{2} \zeta}{\alpha^{2}}\right) S-\frac{m S_{12}}{2}-\frac{\left(S_{1} e^{-\zeta}+S_{2} e^{\zeta}\right) \operatorname{sh} \zeta}{2 \alpha}, \\
& l_{3}=i\left[\frac{1}{2 m}\left(1+\frac{\operatorname{sh}^{2} \zeta}{\alpha^{2}}\right) S+\frac{m S_{12}}{2}+\frac{\left(S_{1} e^{-\zeta}+S_{2} e^{\zeta}\right) \operatorname{sh} \zeta}{2 \alpha}\right],
\end{aligned}
$$

где

$$
\zeta=\frac{1}{2} \ln \left(z_{2} / z_{1}\right)
$$

H

$$
\begin{aligned}
S_{1} & =\frac{\pi m \alpha}{4 \operatorname{sh} \zeta}\left(J_{p 1}^{\prime} N_{p 2}-J_{p 2} N_{p 1}^{\prime}\right), \\
S_{2} & =\frac{\pi m \alpha}{4 \operatorname{sh} \zeta}\left(J_{p 1} N_{p 2}^{\prime}-J_{p 2}^{\prime} N_{p 1}\right), \\
S & =\frac{\pi m \alpha}{4 \operatorname{sh} \zeta}\left(J_{p 1} N_{p 2}-J_{p 2} N_{p 1}\right), \\
S_{12} & =\frac{\pi m \alpha}{4 \operatorname{sh} \zeta}\left(J_{p 1}^{\prime} N_{p 2}^{\prime}-J_{p 2}^{\prime} N_{p 1}^{\prime}\right) .
\end{aligned}
$$

В последних формулах штрих у $J_{p}$ и $N_{p}$ означает производную по $y$, а второй индекс у $J_{p}$ и $N_{p}$ и их производных указывает на аргумент $z_{1}$ или $z_{2}$ соответственно. Очевидно,

$$
S_{1} S_{2}-S S_{12}=1
$$

Подставляя в формулу (9) выражения $l_{0}, l_{1}, l_{2}, l_{3}$ из формул (31), находим элементы матрицы интерференции:

$$
\begin{aligned}
& L_{12,11}^{0}=L_{1222}^{0^{*}}=-\frac{\operatorname{sh} \zeta \operatorname{sh}\left(v_{21}^{0}+\zeta\right)}{m \alpha} \cdot S+\frac{n_{1}^{0} S_{2}-n_{2}^{0} S_{1}}{2 \sqrt{n_{1}^{0} n_{2}^{0}}}+ \\
& +i\left[\frac{\sqrt{n_{1}^{0} n_{2}^{0}}}{2 m}\left(1+\frac{\mathrm{sh}^{2} \zeta}{n_{1}^{0} n_{2}^{0} \alpha^{2}}\right) S+\frac{m S_{12}}{2 \sqrt{n_{1}^{0} n_{2}^{0}}}+\frac{\left(S_{1} e^{-\zeta}+S_{2} e^{\zeta}\right) \operatorname{sh} \zeta}{2 \alpha \sqrt{n_{1}^{0} n_{2}^{0}}}\right], \\
& L_{12,21}^{0}=L_{12,12}^{0^{*}}=\frac{\operatorname{sh} \zeta \operatorname{ch}\left(v_{21}+\zeta\right)}{m \alpha} \cdot S-\frac{n_{1}^{0} S_{2}+n_{2}^{0} S_{1}}{2 \sqrt{n_{1}^{0} n_{2}^{0}}}- \\
& +i\left[\frac{\sqrt{n_{1}^{0} n_{2}^{0}}}{2 m}{ }^{2} 1-\frac{\operatorname{sh}^{2} \zeta}{n_{1}^{0} n_{2}^{0} \alpha^{2}}\right) S-\frac{m S_{12}}{2 \sqrt{n_{1}^{0} n_{2}^{0}}}-\frac{\left(S_{1} e^{-\zeta}+S_{2} e^{\zeta}\right) s h \zeta}{2 \alpha \sqrt{n_{1}^{0} n_{2}^{0}}} .
\end{aligned}
$$

3 ENSV TA Toimetised $F^{*} M-11976$ 
Здесь

$$
v_{21}^{0}=v_{2}^{0}-v_{1}^{0}=\frac{1}{2} \ln \left(n_{2}^{0} / n_{1}^{0}\right) .
$$

В случае отсутствия скачка показателя преломления на границах $n_{1}{ }^{0}$, $n_{2}^{0}$ следует заменить на $n_{1}, n_{2}$ и $v_{21}^{0}$ на

$$
v_{21}=\frac{1}{2} \ln \left(n_{2} / n_{1}\right) \text {. }
$$

Учитывая, что согласно первой формуле (25)

$$
m^{2}=\frac{n_{1} n_{2} \operatorname{sh}\left(2 v_{21}+2 \zeta\right)}{\operatorname{sh} 2 \zeta},
$$

находим для этого случая элементы матрицы интерференции в виде:

$$
\begin{aligned}
& L_{12,11}=L_{12,22}^{*}=-\frac{\operatorname{sh} 2 \zeta \sqrt{\operatorname{th} \zeta \operatorname{th}\left(v_{21}+\zeta\right)}}{2 \alpha \sqrt{n_{1} n_{2}}} \cdot S+\frac{n_{1} S_{2}-n_{2} S_{1}}{2 \sqrt{n_{1} n_{2}}}+ \\
& +i\left[\frac{\sqrt{\operatorname{sh} 2 \zeta}}{2 \sqrt{\operatorname{sh}\left(2 v_{21}+2 \zeta\right)}}\left(1-\frac{\operatorname{sh}^{2} \zeta}{n_{1} n_{2} \alpha^{2}}\right) S+\frac{S_{12} \sqrt{\operatorname{sh}\left(2 v_{21}+2 \zeta\right)}}{2 \sqrt{\operatorname{sh} 2 \zeta}}+\right. \\
& \left.+\frac{\left(S_{1} e^{-\zeta}+S_{2} e^{\zeta}\right) \operatorname{sh} \zeta}{2 \alpha \sqrt{n_{1} n_{2}}}\right], \\
& L_{12,21}=L_{12,12}^{*}=\frac{\operatorname{sh} 2 \zeta \sqrt{\operatorname{th} \zeta \operatorname{cth}\left(v_{21}+\zeta\right)}}{2 \alpha \sqrt{n_{1} n_{2}}} \cdot S+\frac{n_{1} S_{2}+n_{2} S_{1}}{2 \sqrt{n_{1} n_{2}}}+ \\
& +i\left[\frac{\sqrt{\operatorname{sh} 2 \zeta}}{2 \sqrt{\operatorname{sh}\left(2 v_{21}+2 \zeta\right)}}\left(1-\frac{\operatorname{sh}^{2} \zeta}{n_{1} n_{2} \alpha^{2}}\right) S-\frac{S_{12} \sqrt{\operatorname{sh}\left(2 v_{21}+2 \zeta\right)}}{2 \sqrt{\operatorname{sh} 2 \zeta}}-\right. \\
& \left.-\frac{\left(S_{1} e^{-\zeta}+S_{2} e^{\zeta}\right) \operatorname{sh} \zeta}{2 \alpha \sqrt{n_{1} n_{2}}}\right] .
\end{aligned}
$$

Из последней формулы получаем для коэффициента отражения следующее выражение:

$$
\begin{aligned}
& R_{12}=\frac{\left(n_{1} S_{2}+n_{2} S_{1}+\alpha^{-1} S \operatorname{sh} 2 \zeta \sqrt{\operatorname{th} \zeta \operatorname{cth}\left(v_{21}+\zeta\right)}\right)^{2}+}{\left(n_{1} S_{2}-n_{2} S_{1}-\alpha^{-1} S \operatorname{sh} 2 \zeta \sqrt{\operatorname{th} \zeta \operatorname{th}\left(v_{21}+\zeta\right)}\right)^{2}+} \\
& +\left[\frac{\sqrt{\operatorname{sh} 2 \zeta}}{\sqrt{\operatorname{sh}\left(2 v_{21}+2 \zeta\right)}}\left(\sqrt{n_{1} n_{2}}-\frac{s^{2} \zeta}{\alpha^{2} \sqrt{n_{1} n_{2}}}\right) S-\frac{\sqrt{n_{1} n_{2} \operatorname{sh}\left(2 v_{21}+2 \zeta\right)}}{\sqrt{\operatorname{sh} 2 \zeta}} \cdot S_{12}-\right. \\
& +\left[\frac{\sqrt{\operatorname{sh} 2 \zeta}}{\sqrt{\operatorname{sh}\left(2 v_{21}+2 \zeta\right)}}\left(\sqrt{n_{1} n_{2}+} \frac{\operatorname{sh}^{2} \zeta}{\alpha^{2} \sqrt{n_{1} n_{2}}}\right) S+\frac{\sqrt{n_{1} n_{2} \operatorname{sh}\left(2 v_{21}+2 \zeta\right)}}{\sqrt{\operatorname{sh} 2 \zeta}} \cdot S_{12}+\right. \\
& \left.-\alpha^{-1}\left(S_{1} e^{-\zeta}+S_{2} e^{\hbar}\right) \operatorname{sh} \zeta\right]^{2} \\
& \left.+\alpha^{-1}\left(S_{1} e^{-\zeta}+S_{2} e^{5}\right) \operatorname{sh} \zeta\right]^{2} \text {. }
\end{aligned}
$$




\section{Численные примеры и приближенные решения}

Для обоих рассмотренных выше случаев примем одинаковые значения показателей преломления на краях пленки:

$$
\begin{aligned}
& n_{1}=1,4, \\
& n_{2}=1,4 \sqrt{3}=2,425 .
\end{aligned}
$$

В первом случае значения $n(z)$ от выбора $z_{1}$ в формуле (10) не зависят, а во втором случае положим

$$
z_{2} / z_{1}=15 / 13
$$

Согласно формулам (25) тогда

$$
\begin{aligned}
m^{2} & =17,71, \\
\mu^{2} & =10647 / 16,
\end{aligned}
$$

а согласно формулам (8), (32) и (38)

$$
\begin{aligned}
\zeta & =0,07155, \\
v_{1} & =0,16824, \\
v_{2} & =0,44289, \\
v_{21} & =0,27465 .
\end{aligned}
$$
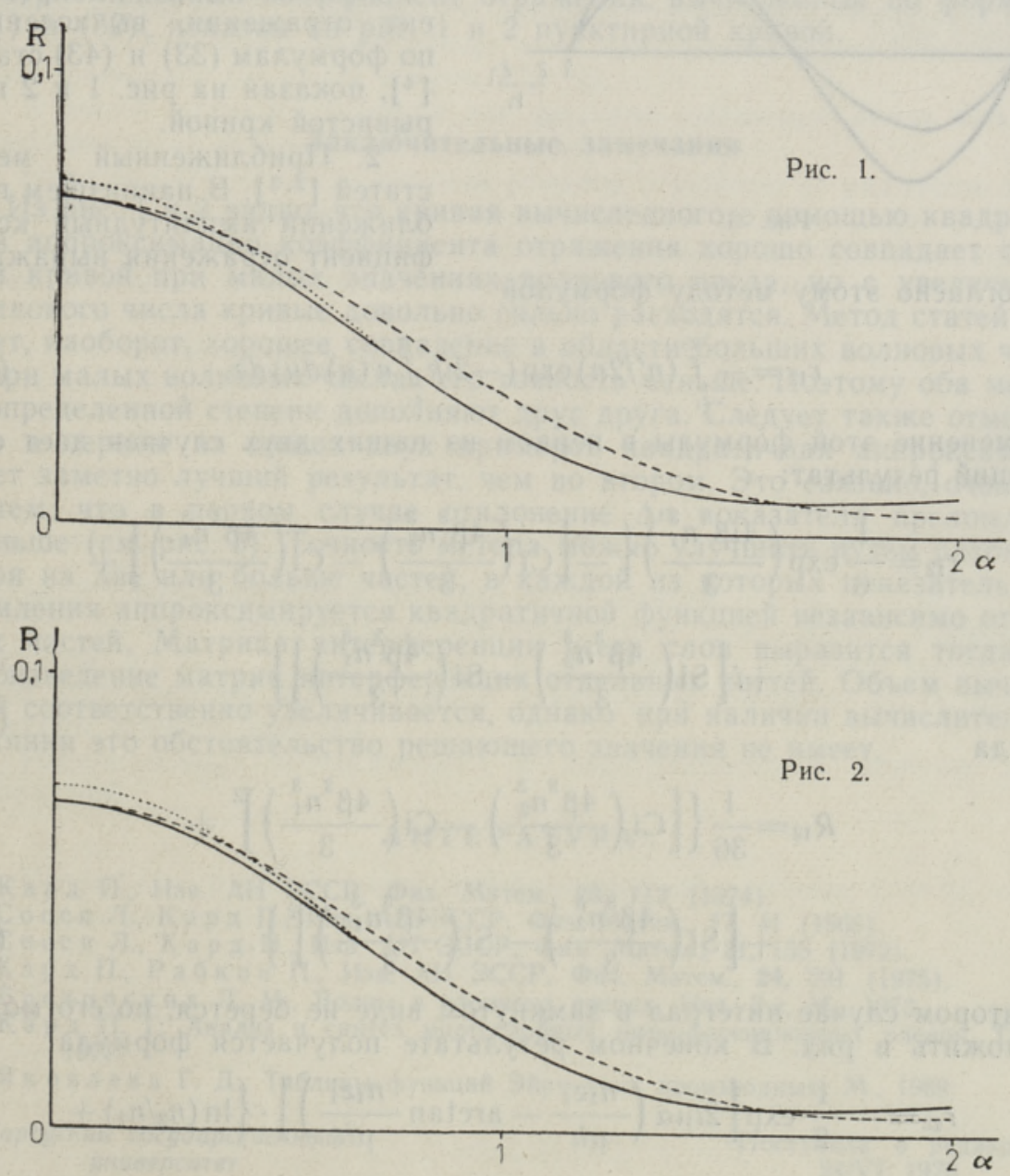
Графики вычисленных по формулам (23) и (42) точных значений коэффициента отражения показаны сплошной кривой на рис. 1 и 2.

Переходим к приближенным решениям.

1. Квадратичная аппроксимация. В первом случае находим (принимая $z_{1}=0$ ) для коэффициентов $A, B, C$ (см. формулу (2)) значения

$$
\begin{aligned}
& A=0,2331, \\
& B=-0,2674, \\
& C=0,7143 .
\end{aligned}
$$

Во втором случае значения этих коэффициентов таковы:

$$
\begin{aligned}
& A=0,2850, \\
& B=-2,1460, \\
& C=16,5704 .
\end{aligned}
$$

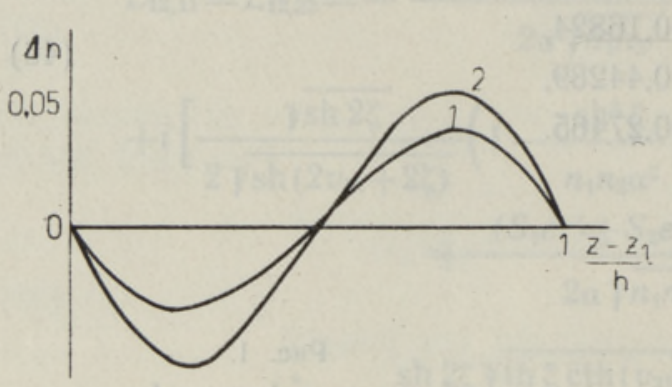

Рис. 3.

ся согласно этому методу формулой

$$
r_{12}=-\int_{z_{1}}^{z_{9}}\left(n^{\prime} / 2 n\right) \exp \left(-2 i k \int_{z_{1}}^{z} n(u) d u\right) d z
$$

Применение этой формулы в первом из наших двух случаев дает следующий результат:

$$
\begin{gathered}
r_{12}=\frac{1}{6} \exp \left(\frac{4 i \beta^{3} n_{1}^{3}}{3}\right)\left\{-\left[\mathrm{Ci}\left(\frac{4 \beta^{3} n_{2}^{3}}{3}\right)-\mathrm{Ci}\left(\frac{4 \beta^{3} n_{1}^{3}}{3}\right)\right]+\right. \\
\left.+i\left[\mathrm{Si}\left(\frac{4 \beta^{3} n_{2}^{3}}{3}\right)-\operatorname{Si}\left(\frac{4 \beta^{3} n_{1}^{3}}{3}\right)\right]\right\}
\end{gathered}
$$

откуда

$$
\begin{aligned}
R_{12} & =\frac{1}{36}\left\{\left[\operatorname{Ci}\left(\frac{4 \beta^{3} n_{2}^{3}}{3}\right)-\operatorname{Ci}\left(\frac{4 \beta^{3} n_{1}^{3}}{3}\right)\right]^{2}+\right. \\
& \left.+\left[\operatorname{Si}\left(\frac{4 \beta^{3} n_{2}^{3}}{3}\right)-\operatorname{Si}\left(\frac{4 \beta^{3} n_{1}^{3}}{3}\right)\right]^{2}\right\} .
\end{aligned}
$$

Во втором случае интеграл в замкнутом виде не берется, но его можно разложить в ряд. В конечном результате получается формула

$$
r_{12}=-\frac{1}{2} \exp \left[2 i \mu \alpha\left(\frac{n_{1} z_{1}}{\mu h}-\arctan \frac{n_{1} z_{1}}{\mu h}\right)\right] \cdot\left\{\ln \left(n_{2} / n_{1}\right)+\right.
$$


$\left.+\sum_{l=1}^{\infty} \frac{\left(-i \alpha \sqrt{n_{1} n_{2}}\right)^{l} \cdot 2^{l+1} \operatorname{ch}^{l} \zeta}{l \cdot l ! 3^{l+1} \operatorname{sh}^{l} 2 v_{21}} \cdot \sum_{p=0}^{\infty} c_{l p} \cdot \frac{\operatorname{sh}^{p} 2 \zeta \operatorname{sh}\left(3 l \zeta+3 l v_{21}+2 p v_{21}\right)}{\operatorname{sh}^{p}\left(2 \zeta+2 v_{21}\right)}\right\}$

где $c_{l p}$ суть численные коэффициенты, значения которых для нескольких первых значений индексов $l$ и $p$ приведены в таблице. Ряд сходится довольно медленно, но удобен для вычислений с помощью ЭВМ.

Таблица чисел $c_{l p}$

\begin{tabular}{c|c|c|c|c|c}
\hline$l$ & \multicolumn{5}{|c}{$p$} \\
\cline { 2 - 6 } & 0 & 1 & 2 & 3 & 4 \\
\hline 1 & 1,00000 & $-0,96000$ & $-0,09061$ & $-0,00853$ & 0,00479 \\
2 & 1,00000 & $-1,65000$ & 0,40029 & 0,15152 & 0,05824 \\
3 & 1,00000 & $-2,29091$ & 1,28535 & 0,10688 & $-0,02578$ \\
4 & 1,00000 & $-2,91429$ & 2,54143 & $-0,37441$ & $-0,18302$ \\
5 & 1,00000 & $-3,52941$ & 4,16232 & $-1,51194$ & $-0,21132$
\end{tabular}

Приближенный коэффициент отражения, вычисленный по формулам (51) и (52), показан на рис. 1 и 2 пунктирной кривой.

\section{Заключительные замечания}

Из рис. 1 и 2 видно, что кривая вычисленного с помощью квадратичной аппроксимации коэффициента отражения хорошо совпадает с точной кривой при малых значениях волнового числа, но с увеличением волнового числа кривые довольно сильно расходятся. Метод статей $\left[{ }^{2,3}\right]$ дает, наоборот, хорошее совпадение в области больших волновых чисел, а при малых волновых числах его точность меньше. Поэтому оба метода в определенной степени дополняют друг друга. Следует также отметить, что в первом из наших двух примеров квадратичная аппроксимация дает заметно лучший результат, чем во втором. Это связано, очевидно, с тем, что в первом случае отклонение $\Delta n$ показателя преломления меньше (см. рис. 3). Точность метода можно улучшить путем разбиения слоя на две или больше частей, в каждой из которых показатель преломления аппроксимируется квадратичной функцией независимо от других частей. Матрица интерференции всего слоя выразится тогда как произведение матриц интерференции отдельных частей. Объем вычислений соответственно увеличивается, однако при наличии вычислительной техники это обстоятельство решающего значения не имеет.

\section{ЛИТЕРАТУРА}

1. К ард П., Изв. АН ЭССР, Физ. Матем., 23, 113 (1974).

2. Сосси Л., Кард П., Изв. АН ЭССР, Физ. Матем., 17, 41 (1968).

3. Сосси Л., К ард П., Изв, АН ЭССР, Физ. Матем., 21, 155 (1972).

4. К а р д П., Р а бкин П., Изв. АН ЭССР, Физ. Матем., 24, 291 (1975).

5. Бреховских Л. М., Волны в слонстых средах, Изд. 2-е, М., 1973.

6. Кард П. Г. Анализ и синтез многослойных интерференционных пленок, Таллин, 1971.

7. Як овле в а Г. Д., Таблицы функций Эйри и их производных, М., 1969. 
P. KARD, H. IHER, J. LEMBRA

\section{UUS MITTEHOMOGEENSE OPTILISE KIHI ANALUUSI LÄHISMEETOD}

Kui mittehomogeense optilise kihi murdumisnäitaja pöördväärtus on koordinaadi ruutfunktsioon, siis avalduvad kihi peegeldumis- ja läbilaskvustegur elementaarfunktsioonide kaudu. Seda asjaolu võib kasutada mistahes kihi ligikaudseks analüüsimiseks (s. o. kihi optiliste karakteristikute arvutamiseks lainepikkuse funktsioonina), lähendades kihi murdumisnäitaja pöördruutfunktsiooniga. Kahe näite varal (valemid (10) ja (24)) selgub, et see meetod on küllalt efektiivne juhul, kui lainearvu väärtused on väikesed.

P. KARD, H. IHER, J. LEMBRA

\section{A NEW APPROXIMATE METHOD FOR THE ANALYSIS OF AN INHOMOGENEOUS OPTICAL FILM}

If the refractive index of an inhomogeneous optical film is an inverse square function of co-ordinate, then the reflectance and the transmittance of the film can be expressed in terms of elementary functions. This circumstance can be used for an approximate analysis of any inhomogeneous film, i. e. for finding its optical characteristics versus wave-length. For that purpose the refractive index of the film must be approximated by an inverse square function. By two instances (formulae (10) and (24)) it is shown that the discussed method is accurate enough, provided the wave number is sufficiently small. 